\title{
Editorial
}

\section{Searching for Meaningful Differences in Viscosity}

The preceding article by Garcia, Chambers, Matta and Clark [1] exposes a serious flaw in our pervasive assumptions about texture modification as an intervention for dysphagia. Namely, Garcia et al. have successfully demonstrated that following a recipe and combining a prescribed amount of thickening agent with a prescribed volume of liquid does not necessarily result in a texture-modified product with predictable flow properties. The data presented by Garcia et al. [1] clearly illustrate that the final product depends on the nature of the selected thickening agent, the nature of the original thin liquid, and time. These factors are shown to significantly influence the viscosity of the final product, even in a controlled laboratory setting. The clarity of Garcia et al.’s [1] findings compels us to take notice, and consider the implications of this research for daily clinical practice.

In fact, Garcia and colleagues [1] are not the first to report this phenomenon in the dysphagia literature. In 1997, Pelletier [2] used five different commercially available thickening powders to mix 4-oz samples of three different liquids (apple-juice, $2 \%$ milk and black coffee) to resemble maple-syrup, honey and pudding, using the manufacturer's directions. Perceptual ratings of taste and consistency were made by a panel of raters using a 3-point scale ranging from poor to good at 1 minute, 5 minutes and 17 hours post mixing. Pelletier's results [2] showed that the judges perceived differences in the viscosity of each liquid-thickener combination at all time points, and that no single thickening agent reliably achieved a judgement of "good" in its ability to match the consistency of maple syrup, honey or vanilla pudding when mixed with apple juice. 
Garcia et al. [1] have improved upon the methodology used by Pelletier [2], (particularly in their use of temperature control and instrumentation to measure viscosity), yet their ultimate findings are surprisingly similar. Three of the five thickening agents (Thick \& Easy ${ }^{\circledR}$, Thick It ${ }^{\circledR}$, and Thicken $\mathrm{Up}{ }^{\circledR}$ ) and three of the five liquids (apple juice, 2\% milk and coffee) used in Garcia et al's study [1] were identical to those studied by Pelletier [2]. In contrast to Pelletier [2], Garcia and colleagues [1] compared the resulting viscosity of their products to viscosity ranges proposed by the National Dysphagia Diet [3] for nectar-thick and honey-thick consistencies, (i.e., 51-350 and 351-1750 centipoise (cP) respectively, at a shear-rate of 50 reciprocal seconds). Their measurements were made at the recipe-prescribed "standard" time-to-thicken, and at 10and 30-minutes post mixing. The findings reveal a confusing and alarming variety of viscosity measurements. At the label-prescribed time-to-thicken, four of the 25 nectar-like products were found to have viscosities in the thin liquid range (less than $50 \mathrm{cP}$ ); by 10-minutes post mixing, two of the 25 nectar-like liquids had viscosities in the honey-liquid range (more than $351 \mathrm{cP}$ ); and by 30-minutes, two additional nectar-thick samples had reached a honey-like viscosity. For the honey-thick liquids, the three measurement intervals respectively identified only 8, 9 and 10 of the 25 samples to be within the targeted National Dysphagia Diet range of 351-1750 cP. At the first measurement interval (standard time-to-thicken), all the other honey-thick samples showed thinner than desirable viscosities in the nectar-thick range. By 10-minutes postthickening, 1 sample had thickened to a spoon-thick viscosity (i.e. more than $1750 \mathrm{cP}$ ), and by 30-minutes, this was true of 3 additional samples.

"Thickening liquids has been and continues to be one of the most frequently used compensatory interventions in hospitals and long-term care facilities” [4]. Yet, our literature contains very few studies to support this wide-spread intervention. It might surprise some readers 
to learn that the available literature contains no evidence to suggest that texture modification reliably reduces aspiration or facilitates improved bolus clearance. Rather, the literature suggests that the influence of increased viscosity can be distilled primarily to temporal effects (increased durations of oral and pharyngeal transit [5, 6], time to peak lingual pressure[7], tongue-base to posterior-wall contact $[5,6]$ and UES-opening $[5,6])$ with scattered additional findings of increased amplitudes in lingual force [8], lingual pressure generation [7, 9], and the range of upward hyoid movement [10, 11]. Yet, by far the majority (if not all) of these studies do not adequately define the viscosities of the texture-modified stimuli studied [12], and in many, the observed effects of viscosity are likely to be confounded by the density and flavour influences of barium [13-15].

To date, our literature has not determined the magnitude of the minimally meaningful difference in viscosity, namely how large a difference in viscosity is required to elicit altered swallowing physiology? Previous literature suggests that (at least at a perceptual level) the answer to this question may itself be a sliding scale. At the thinner end of the viscosity continuum, we are able to detect small changes in viscosity [16], and these might be sufficient to influence lingual bolus control or swallow timing. At the other end of the spectrum, however, we are much less sensitive to increases in the viscosity of thicker substances, and may need differences of much greater magnitude to elicit differences in swallowing behaviours [16]. However, several papers suggest that healthy individuals do not, in fact, show viscositydependent modulations in tongue movements, lingual pressures or hyoid movement across stimuli within the thin to honey-thick viscosity range $[17,18]$. Whether dysphagic individuals are more likely to exhibit differences in swallowing in responses to viscosity differences of smaller magnitudes remains an as-yet unanswered empirical question. 
Both Pelletier [2] and Garcia et al. [1] have demonstrated time-dependency and productdependent variability in the viscosity of thickened liquids under controlled circumstances, using carefully prescribed recipes. What should we make of these results? Does this inherent variability suggest that the widely presumed benefits of texture modification are a figment of our collective imagination? Certainly for patients who appear to lose control of thin liquids, providing a more viscous bolus makes good, logical sense. Conversely, for patients who exhibit difficulty with oropharyngeal transport of thicker substances like mashed potato, thinning the bolus is a logical and pragmatic intervention. However, from Garcia et al.'s data [1], it is clear that using set amounts of thickening agent with different liquids will not reliably yield endproducts of equivalent viscosity. In Garcia et al.'s study [1], the magnitude of the observed viscosity variations across liquids ranges from $40-353 \mathrm{cP}$ for nectar-thick liquids and from 114 to $3395 \mathrm{cP}$ for the honey-thick liquids. It is notable that the magnitude of this variation exceeds the proposed size of entire centipoise-defined classification ranges within the National Dysphagia Diet [3] (i.e. $300 \mathrm{cP}$ for the nectar-thick class and $1400 \mathrm{cP}$ for the honey-thick class). We should also take serious note of Garcia et al.'s [1] evidence of time-dependent increases in the viscosity of liquids thickened with commercially available thickening agents. By 30-minutes post mixing, all liquids mixed with starch-based thickeners displayed increases in viscosity compared to the standard time-to-thicken measurement, with these increases ranging from 14 to $296 \mathrm{cP}$ within the nectar-thick group and from 85 to more than $4000 \mathrm{cP}$ within the honey-thick group. By contrast, liquids mixed with the gum-based thickening agents showed relatively stable viscosities across time, with slight thinning evident by 30 minutes (- 26 to $+4 \mathrm{cP}$ for the nectar-thick samples and 18 to $+3 \mathrm{cP}$ for the honey-thick samples). Without data from viscosity measurements at further intervals, we cannot speculate regarding the time necessary for the starch-thickened liquids to 
achieve stability, nor whether further thinning would be likely within the gum-thickened liquids with additional time.

Garcia et al.'s findings [1] do suggest that it is unreasonable to expect manufacturers to define recipes that will produce similar products across all liquids. In defense of the manufacturers, it may also be unfair of us to evaluate their recipes using the National Dysphagia Diet guidelines for liquid viscosities, since the published recipes on product labels may well predate those guidelines, and the guidelines themselves are acknowledged to be a consensus-based proposal rather than an evidence-based criterion [3]. Beyond the clinical gut level of eye-balling thickness, and estimating when a liquid is "just right", "too thin", or "too thick" for any individual patient, it seems we have relatively little empirical evidence to guide us. Although the magnitude of the observed viscosity variations in Garcia's data [1] is impressive, and certainly sufficient to make us question the validity both of thickening recipes and of the classification boundaries proposed within the National Dysphagia Diet [3], whether this variation is of sufficient magnitude to warrant real concern for our patients remains unclear. It appears that the question we need to address in future research, is that of defining the minimally interesting or minimally meaningful difference in viscosity for oropharyngeal swallowing physiology.

Catriona M. Steele, Ph.D., SLP(C), CCC-SLP, Reg. CASLPO

Scientist and Corporate Practice Leader for Speech-Language Pathology and Audiology, Toronto Rehabilitation Institute

Assistant Professor,

Graduate Department of Speech-Language Pathology, University of Toronto 
550 University Avenue, \#801

Toronto, ON, M5G 2A2

e-mail: steele.catriona@torontorehab.on.ca

1. Garcia JM, Chambers E, Matta Z, and Clark M: Viscosity measurements of nectar and honey-thick liquids: Product, liquid and time comparisons. Dysphagia (this issue), 2005.

2. Pelletier CA: A comparison of consistency and taste of five commercial thickeners. Dysphagia 12(2):74-78, 1997.

3. National Dysphagia Diet Task Force ADA: National dysphagia diet: Standardization for optimal care, ed. Clayton J. Chicago, IL: Faulhaber, D, 2002

4. Robbins J, et al.: Defining physical properties of fluids for dysphagia evaluation and treatment. Perspectives on Swallowing and Swallowing Disorders (Newsletter of the American Speech-Language Hearing Association Special Interest Division 13) 11(2):1619, 2002.

5. Bisch EM, Logemann JA, Rademaker AW, Kahrilas PJ, and Lazarus CL: Pharyngeal effects of bolus volume, viscosity, and temperature in patients with dysphagia resulting from neurologic impairment and in normal subjects. J Speech Hear.Res. 37(5):10411059, 1994.

6. Lazarus CL, et al.: Effects of bolus volume, viscosity, and repeated swallows in nonstroke subjects and stroke patients. Archives of Physical Medicine and Rehabilitation 74(10):1066-1070, 1993.

7. Pouderoux P and Kahrilas PJ: Deglutitive tongue force modulation by volition, volume, and viscosity in humans. Gastroenterology. 108(5):1418-1426, 1995.

8. Miller JL: The influence of volume and viscosity on the distribution of anterior lingual force during oral stage swallowing. Masters thesis. School of Communication Sciences and Disorder, McGill University: Montreal. 1993.

9. Nicosia MA, et al.: Age effects on the temporal evolution of isometric and swallowing pressure. Journals of Gerontology Series A-Biological Sciences \& Medical Sciences. 55(11):M634-640, 2000.

10. Perlman AL, Vandaele DJ, and Otterbacher MS: Quantitative assessment of hyoid bone displacement from video images during swallowing. J Speech Hear. Res. 38(3):579-585, 1995.

11. Ishida R, Palmer JB, and Hiiemae KM: Hyoid motion during swallowing: Factors affecting forward and upward displacement. Dysphagia 17(4):262-272, 2002.

12. Cichero JAY, Hay G, Murdoch BE, and Halley PJ: Videofluoroscopy fluids versus mealtime fluids: Differences in viscosity and density made clear. Journal of Medical Speech Language Pathology 5:203-215, 1997.

13. Mills RH: Rheology overview: Control of liquid viscosities in dysphagia management. Nutrition in Clinical Practice 14:S52-S56, 1999.

14. Steele CM and Van Lieshout PHHM: Does barium influence tongue behaviors during swallowing? American Journal of Speech-Language Pathology 14(1):27-39, 2005. 
15. Dantas RO, Dodds WJ, Massey BT, and Kern MK: The effect of high- vs low-density barium preparations on the quantitative features of swallowing. AJR: American Journal of Roentgenology 153(6):1191-1195, 1989.

16. Smith CH, Logemann JA, Burghardt WR, Carrell TD, and Zecker SG: Oral sensory discrimination of fluid viscosity. Dysphagia 12(2):68-73, 1997.

17. Steele CM and Van Lieshout PHHM: The influence of bolus consistency on lingual behaviors in sequential swallowing. Dysphagia 19(3):1-11, 2004.

18. Chi-Fishman G and Sonies BC: Effects of systematic bolus viscosity and volume changes on hyoid movement kinematics. Dysphagia 17(4):278-287, 2002. 